\title{
Oxygen matters: hypoxia as a pathogenic mechanism in rhinosinusitis
}

\author{
Hyung-Ju Cho ${ }^{1,2}$ E Chang-Hoon Kim ${ }^{1,2, *}$ \\ ${ }^{1}$ Department of Otorhinolaryngology, ${ }^{2}$ The Airway Mucus Institute, Yonsei University College of Medicine, Seoul 03722, Korea
}

The airway epithelium is the first place, where a defense mechanism is initiated against environmental stimuli. Mucociliary transport (MCT), which is the defense mechanism of the airway and the role of airway epithelium as mechanical barriers are essential in innate immunity. To maintain normal physiologic function, normal oxygenation is critical for the production of energy for optimal cellular functions. Several pathologic conditions are associated with a decrease in oxygen tension in airway epithelium and chronic sinusitis is one of the airway diseases, which is associated with the hypoxic condition, a potent inflammatory stimulant. We have observed the overexpression of the hypoxia-inducible factor 1 (HIF-1), an essential factor for oxygen homeostasis, in the epithelium of sinus mucosa in sinusitis patients. In a series of previous reports, we have found hypoxia-induced mucus hyperproduction, especially by MUC5AC hyperproduction, disruption of epithelial barrier function by the production of VEGF, and down-regulation of junctional proteins such as ZO-1 and E-cadherin. Furthermore, hypoxia-induced inflammation by HMGB1 translocation into the cytoplasm results in the release of IL-8 through a ROS-dependent mechanism in upper airway epithelium. In this mini-review, we briefly introduce and summarize current progress in the pathogenesis of sinusitis related to hypoxia. The investigation of hypoxia-related pathophysiology in airway epithelium will suggest new insights on airway inflammatory diseases, such as rhinosinusitis for clinical application and drug development. [BMB Reports 2018; 51(2): 59-64]

\section{INTRODUCTION}

The airway epithelium constitutes the first line of defense against environmental stimuli, in innate immunity by serving

*Corresponding author. Tel: +82-2-2228-3609; Fax: +82-2-3930580; E-mail: entman@yuhs.ac

https://doi.org/10.5483/BMBRep.2018.51.2.014

Received 27 December 2017

Keywords: Airway, Epithelial junction, Epithelium, HIF-1 $\alpha$, Hypoxia, Innate immunity, Mucin, Rhinosinusitis, VEGF as mechanical barriers along with the mucociliary transport (MCT), which is the defense mechanism of the airway (1). Preserving this physiologic role requires sustaining energy production, which is supplied by appropriate oxygenation (2). Several pathologic conditions may lead to decrease in oxygen level in the airway epithelium. In chronic airway diseases such as sinusitis, allergic rhinitis, asthma, and chronic obstructive pulmonary disease, decrease in oxygen tension can occur due to pathologic changes in the microvascular structures or increase in metabolic demands (3). Such diseases commonly represent pathologic characteristics such as infiltration of inflammatory cells, tissue remodeling or mucus hypersecretion (4).

In the upper airway, chronic sinusitis is one of the hypoxia-related diseases. The sinus mucosa is composed of ciliated columnar epithelium intermixed with goblet cells. The cilia of epithelial cells have an essential role in transporting mucus outside of sinus through sinus ostium and maintaining a normal physiologic condition in the paranasal sinuses. Normal mucociliary transport is essentialfor the maintenance of the airway innate defense, and a decrease of mucociliary transport has been proved in rhinosinusitis. The defect in normal mucociliary transport can develop due to changes in mucus viscosity or any toxicity (5). Hypoxia is another potential factor for sinusitis and we have reviewed the pathogenesis of hypoxia-related sinusitis.

\section{HIF-1 $\alpha$-MEDIATED MUCUS HYPERSECRETION BY HYPOXIA}

The mechanical obstruction of the sinus natural opening reduces oxygen tension within the sinus resulting in sinusitis (6). Goblet cell hyperplasia is one of the major histopathologic changes in chronic rhinosinusitis (7). In hypoxic conditions, the hypoxia-inducible factor 1 (HIF-1) is essential for oxygen-related pathophysiology for transcriptional expression of erythropoietin (8), vascular endothelial growth factor (VEGF) (9), heme oxygenase-1 (10), and transferrin (11). HIF-1 is composed of a heterodimer, $\alpha$ and $\beta$ subunits (12), and HIF- $1 \alpha$ activation conducts the extracellular signal-regulated kinase signaling pathway (13). Although hypoxia is an effective inflammatory stimulant (4), the influence of hypoxia on mucus overproduction and its related-mechanism has not been 
clearly suggested. The hypoxia-response element (HRE) is commonly present at the proximal promoter and includes one or more HIF-1-binding sites (14). The mutation in the HRE location inactivates the transcriptional response to hypoxia $(15,16)$. The promoter region of the MUC5AC gene includes a similar sequence to the $\operatorname{HRE}(17,18)$. Therefore, we investigated the promoter region of the MUC5AC gene to understand the mechanism of hypoxia-induced MUC5AC gene in airway epithelium. We have mainly utilized primary human nasal epithelial (HNE) cells, which were cultured and differentiated in the condition of the air-liquid interface system, for in vitro experiments (19). Under hypoxic condition, HNE cells induced the expression of MUC5AC mRNA and protein (20). The elevation of HIF- $1 \alpha$ expression in HNE cell by hypoxia was also identified and its loss- or gain-of-function experiment confirmed the role of HIF-1 $\alpha$ in MUC5AC expression under hypoxic environment. To identify the DNA binding activity of HIF- $1 \alpha$ to the MUC5AC promoter under hypoxia, we performed chromatin immunoprecipitation (ChIP) assays and the regulatory HRE region of the MUC5AC promoter was identified to be important in increasing the transcriptional activity of MUC5AC caused by hypoxia (20). Immunohistochemical staining proved strong expression of MUC5AC and HIF- $1 \alpha$ in the epithelium in sinusitis mucosa. These data suggest that hypoxic condition in the sinus is associated with sinusitis in terms of MUC5AC overproduction via the HIF-1 $\alpha$-mediated mechanism.

\section{EPITHELIAL BARRIER BY HYPOXIA: VEGF MEDIATED-MECHANISM}

The pathophysiology of epithelial disruption have been studied based on several aspects. It was demonstrated that hypoxia contributed to the breakdown of epithelial barrier via VEGFR-1 in retinal epithelium (21). The IL-13 led to disruption of tight junction in bronchial epithelium (22). Rhinovirus infection is a fundamental predisposing factor for subsequent bacterial invasion by dissociating zona occludens-1 (23). To the best of our knowledge, this is the first report to elucidate the role of the hypoxia-HIF-VEGF axis in the regulation of epithelial paracellular permeability in airway epithelium.

VEGF is a protein associated with vascular permeability as well as angiogenesis in endothelial cells and induces fenestrations or caveolae in the endothelial cytoplasm, which result in a vascular leak of plasma protein and tissue edema (24). There exist several reports on hypoxia-induced VEGF secretion (25-27). Furthermore, VEGF overexpression has been reported in several chronic airway inflammatory diseases, such as bronchial asthma, sinusitis, and allergic rhinitis (28-31). Therefore, it would be interesting to understand the role of VEGF in the pathogenesis of sinusitis under hypoxia. We hypothesized that HIF- $1 \alpha$ and VEGF might be associated with the pathogenesis of sinusitis by augmenting paracellular permeability in the sinus epithelium. In human airway epithelial cells, the elevation in VEGF mRNA and protein was identified by hypoxic stimulation; overexpression of HIF- $1 \alpha$ under normoxic condition also induced VEGF expression (32). Knockdown of HIF-1 $\alpha$ resulted in downregulation of VEGF mRNA and protein level under hypoxic condition. These results imply that VEGF expression under the hypoxic condition is mediated through HIF- $1 \alpha$ signaling pathway. The functional assay of the epithelial barrier can be measured by transepithelial electrical resistance (TEER). TEER decreases under the hypoxic condition and can be rescued by the knockdown of HIF-1 $\alpha$ or bevacizumab, a monoclonal antibody against VEGF. However, we have not yet confirmed the expression of VEGFR-1 or -2 in primary nasal epithelial cells and further investigations are required to elucidate the signaling pathway including identification of VEGFR.

Disruption of epithelial barrier function is an important histological change resulting in clinical significance. This fact provides a possibility of the development of novel therapeutic agents to improve epithelial barrier function for various airway diseases. Vulnerability to adherence or invasion of pathogens can be increased through the leaky epithelial barrier. We also confirmed higher bacterial passage of nasal epithelium under hypoxic condition compared to normoxia.

\section{EPITHELIAL BARRIER BY HYPOXIA: JUNCTIONAL PROTEINS MEDIATED-MECHANISM}

The epithelial barrier function is maintained by tight and adherence junctions. The tight junctions are the most apical complex and disconnect the apical lumen from the basolateral side. ZO-1 is a tight junction component that is present in the upper part of epithelium (33). Adherence junctions are also important for the intercellular adhesion as they provide a docking site for signaling molecules $(34,35)$. The main component of adherence junction is E-cadherin, a transmembrane protein that forms calcium-dependent hemophilic intercellular adhesions between epithelial cells (36). In the human nasal mucosa, viral infection leads to loss of junctional complexes, especially ZO-1 with resultant augmentation of intranasal bacterial inoculation in mice (23). In allergic nasal mucosa, the reduction of ZO-1 mRNA has been reported (37). The reduction of ZO-1 but the elevation of E-cadherin was observed in the epithelium of nasal polyp (38). Therefore, the alterations in the levels of ZO-1 or E-cadherin can cause the disruption of the epithelial barrier under various pathological conditions (39).

To prove the effect of hypoxia on barrier function, we investigated the effects of hypoxia on ZO-1 and E-cadherin expression level (40). Hypoxia decreased the expression of ZO-1 and E-cadherin significantly after 8 hours of exposure. The disruption of the epithelial barrier was also proven functionally based on the measurement of TEER. The decreased expression pattern of ZO-1 and E-cadherin was also confirmed in the sinus epithelium of chronic sinusitis which is 
a hypoxia-conditioned nasal mucosa. Overall, the exposure to hypoxic conditions cause down-regulation of junction complex molecules and increasing TEER implying the disruption of normal barrier function of nasal epithelia.

\section{HMGB1-MEDIATED INFLAMMATION BY HYPOXIA}

High-mobility group box 1 (HMGB1) is a small sized protein, which acts as a DNA chaperone. HMGB1 is secreted into the extracellular space either actively or passively. Release following pro-inflammatory stimulation is an active process and release following apoptosis and necrosis is a passive process. The HMGB1, which is released into the extracellular space, binds to Toll-like receptor (TLR) 2 or TLR 4, and the receptor for advanced glycation end products (RAGE) resulting in activation of proinflammatory signaling pathways (41-43). The HMGB1, which is translocated from the nucleus into the cytoplasm, can be dependent on the posttranslational modifications such as phosphorylation, acetylation, and oxidation; reactive oxygen species (ROS) play important role in this process $(41,42,44,45)$. Recently, we reported the detection of elevated levels of HMGB1 in the nasal lavage fluid collected from chronic rhinosinusitis patients (46). Under hypoxic condition, there exists the possibility that HMGB1 can be translocated from the nucleus into the cytoplasm and released into the extracellular space, thus serving as a characteristic molecule for hypoxia-associated tissue damage $(47,48)$. Therefore, we investigated the role of HMGB1 in the progression of upper airway inflammatory diseases under hypoxic condition. Hypoxia induces translocation of HMGB1 from the nucleus into the extracellular area in RPMI 2650 cells and HNE cells. Immunofluorescence assay (ELISA and western blotting) revealed augmentation of cytoplasmic HMGB1 under the hypoxic condition and increased the level of HMGB1 protein in supernatants from HNE cells (49). Hypoxia increases the redox potential of local environment due to alterations in the production of ROS (50). ROS production can be dependent on the oxygen tension because the mild degree of hypoxia induces ROS production, but severe hypoxia decreases ROS production (50). The variations in oxygen concentration differentially affect the redox potential of the HMGB1 structure thus altering the functions of HMGB1. In our experimental setting, hypoxia increased the ROS levels significantly, which was confirmed by the pretreatment of the ROS scavenger, $\mathrm{N}$-acetyl cysteine (NAC) with resultant suppression of hypoxia-induced ROS levels. Immunofluorescence assay revealed a reduction in cytoplasmic translocation of HMGB1, implying the association of HMGB1 with the increase in ROS.

Extracellular secretion of HMGB1 was also determined and pretreatment with NAC decreased the HMGB1 protein level from collected apical supernatants by ELISA and western

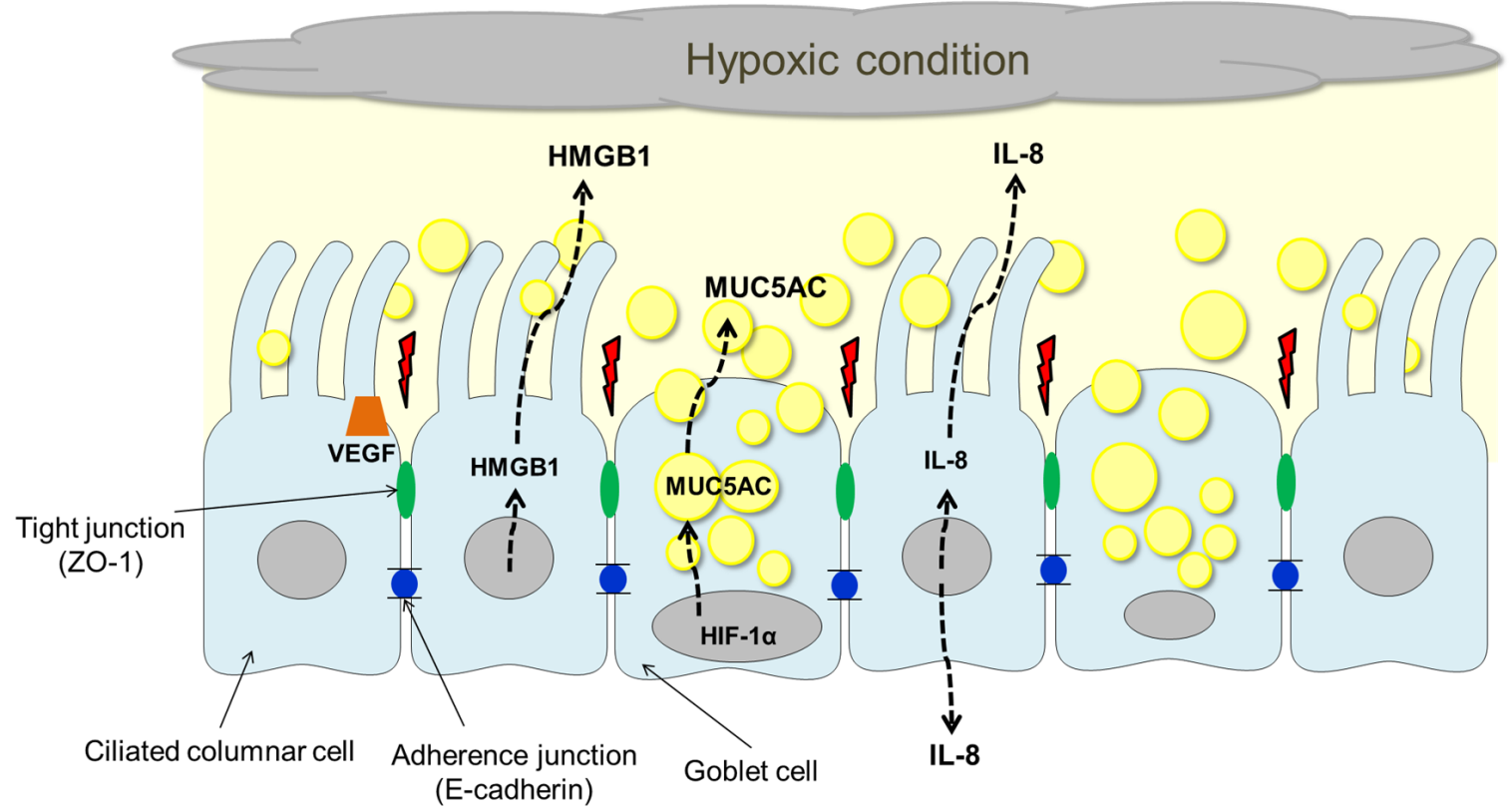

Fig. 1. Hypoxia-induced pathophysiology of upper airway inflammation. Under hypoxic condition, HIF- $1 \alpha$ serves as an essential factor mediating MUC5AC overproduction. Hypoxia stimulates HIF-1 $\alpha$-dependent overexpression of VEGF leading to disruption of epithelial barrier function and alteration in adherence (E-cadherin) or junctional (ZO-1) protein. Furthermore, hypoxia induces HMGB1 translocation into the cytoplasm and release of IL-8 through a ROS-dependent mechanism in the airway epithelium. 
blotting (49). NADPH oxidase enzymes can generate ROS and dual oxidase (DUOX) 1 and 2. The subtypes of NADPH oxidase enzymes play an important role in the production of ROS in airway inflammation (51). Knockdown of DUOX 2 gene using short hairpin RNA showed (shDUOX2) decreased ROS production in HNE cells but no changes were reported in knockdown of DUOX 1 gene. The shDUOX2-transfected HNE cells also showed a reduction in HMGB1 secretion under hypoxic condition (49). Taken together, it is apparent that DUOX2 but not DUOX1 is essential in hypoxia-induced HMGB1 secretion and DUOX2 can lead to ROS-mediated activation of TLR2 and TLR4 in the upper airway epithelium (51).

The nasal secretions of chronic rhinosinusitis patients may contain mast cell tryptase, neutrophil elastase, eosinophil cationic protein, nitric oxide metabolites, IL-1, IL-5, or IL-8 suggesting that these molecules are involved in the development of chronic inflammation in the upper airway (52-55). HMGB1 binds to several specific cell surface receptors, such as the RAGE, TLR, and act as a cytokine-like protein inducing chemotaxis and cytokine release. We determined HMGB1, TNF- $\alpha$, IL-1 $\beta$, and IL- 8 in the nasal secretions of chronic rhinosinusitis patients and performed correlation analysis with the Lund-Mackay score, which is a scoring system indicating the severity of sinonasal symptoms. TNF- $\alpha$ was detected in only $21 \%$ and IL- $1 \beta$ was detected in $44 \%$ of patients with no correlation with symptom severity (46). However, both HMGB1 and IL-8 were detected in all nasal lavage samples from patients and significant correlation was observed with the severity of Lund-Mackay score. Interestingly, the level of HMGB1 was significantly correlated with the level of IL-8 (46). Therefore, we investigated IL-8 in HNE cells under hypoxia and found that IL-8 secretion was increased by hypoxia and suppressed by NAC pretreatment. This finding implies that IL-8 secretion can be dependent on ROS signaling pathway. Furthermore, the treatment of HMGB1 using mammalian recombinant HMGB1 (rHMGB1) induced IL-8 secretion in apical culture supernatants. During the course of employment of anti-HMGB1 blocking antibody to inhibit the function of the secreted HMGB1 protein, IL-8 production was abrogated (49). This observation is very interesting because HMGB1 may be associated with specific cytokines such as IL-6, IL-8, and IL-33 in the nasal epithelium $(56,57)$. HMGB1 also induces the release of IL-1 $\alpha$, IL-1 $\beta$, IL-6, IL- 8 , and TNF- $\alpha$ in macrophages and TNF- $\alpha$, IL-1 $\beta$, and IL- 8 in neutrophils (58). In endothelial cells, HMGB1 can increase the production of tissue factor, an initial protein involved in the coagulation cascade and regulation of fibrinolysis $(59,60)$.

\section{CONCLUSION}

Analysis of the results of previously reported studies indicated that hypoxia plays an important role in the pathogenesis of upper airway inflammation, especially in the chronic rhinosinusitis (Fig. 1). HIF-1 $\alpha$ is an essential factor for oxygen homeostasis in the epithelium and mediates MUC5AC overproduction. HIF- $1 \alpha$ mediated VEGF overexpression and alteration of junctional proteins such as ZO-1 and E-cadherin are also important aspects leading to the disruption of the epithelial barrier under hypoxic condition. Furthermore, hypoxia induces HMGB1 translocation into the cytoplasm and release of IL-8 through a ROS-dependent mechanism in the airway epithelium. It is hypothesized that the investigation of hypoxia-related pathophysiology in airway epithelium will suggest a novel therapeutic target for upper airway inflammatory diseases.

\section{ACKNOWLEDGEMENTS}

This work was supported by the National Research Foundation of Korea (NRF), Grant funded by the Korean Government (MSIP) (No. 2016R1A5A2008630), and by the Bio \& Medical Technology Development Program of the National Research Foundation (NRF) funded by the Ministry of Science, ICT \& Future Planning (NRF-2016M3A9D5A01952414).

\section{CONFLICTS OF INTEREST}

The authors have no conflicting interests.

\section{REFERENCES}

1. Zabner J, Winter M, Excoffon KJ et al (2003) Histamine alters E-cadherin cell adhesion to increase human airway epithelial permeability. J Appl Physiol 95, 394-401

2. Jain M and Sznajder JI (2005) Effects of hypoxia on the alveolar epithelium. Proc Am Thorac Soc 2, 202-205

3. Bunn HF and Poyton RO (1996) Oxygen sensing and molecular adaptation to hypoxia. Physiol Rev 76, 839-885

4. Steinke JW, Woodard CR and Borish L (2008) Role of hypoxia in inflammatory upper airway disease. Curr Opin Allergy Clin Immunol 8, 16-20

5. Lok CN and Ponka P (1999) Identification of a hypoxia response element in the transferrin receptor gene. J Biol Chem 274, 24147-24152

6. Matsune $S$, Kono $M$, Sun D, Ushikai $M$ and Kurono $Y$ (2003) Hypoxia in paranasal sinuses of patients with chronic sinusitis with or without the complication of nasal allergy. Acta Otolaryngol 123, 519-523

7. Rose MC and Voynow JA (2006) Respiratory tract mucin genes and mucin glycoproteins in health and disease. Physiol Rev 86, 245-278

8. Semenza GL and Wang GL (1992) A nuclear factor induced by hypoxia via de novo protein synthesis binds to the human erythropoietin gene enhancer at a site required for transcriptional activation. Mol Cell Biol 12, 5447-5454

9. Levy AP, Levy NS, Wegner S and Goldberg MA (1995) Transcriptional regulation of the rat vascular endothelial growth factor gene by hypoxia. J Biol Chem 270, 13333-13340

10. Lee PJ, Jiang BH, Chin BY et al (1997) Hypoxia-inducible 
factor-1 mediates transcriptional activation of the heme oxygenase- 1 gene in response to hypoxia. J Biol Chem 272, 5375-5381

11. Rolfs A, Kvietikova I, Gassmann M and Wenger RH (1997) Oxygen-regulated transferrin expression is mediated by hypoxia-inducible factor-1. J Biol Chem 272, 2005520062

12. Wang GL and Semenza GL (1995) Purification and characterization of hypoxia-inducible factor 1. J Biol Chem 270, 1230-1237

13. Neruntarat $C$ (2003) Uvulopalatal flap for snoring on an outpatient basis. Otolaryngol Head Neck Surg 129, 353-359

14. Semenza GL (1998) Hypoxia-inducible factor 1 and the molecular physiology of oxygen homeostasis. J Lab Clin Med 131, 207-214

15. Semenza GL, Jiang BH, Leung SW et al (1996) Hypoxia response elements in the aldolase $A$, enolase 1 , and lactate dehydrogenase A gene promoters contain essential binding sites for hypoxia-inducible factor 1 . J Biol Chem 271, 32529-32537

16. Forsythe JA, Jiang $\mathrm{BH}$, lyer NV et al (1996) Activation of vascular endothelial growth factor gene transcription by hypoxia-inducible factor 1 . Mol Cell Biol 16, 4604-4613

17. Li D, Gallup M, Fan N, Szymkowski DE and Basbaum CB (1998) Cloning of the amino-terminal and 5'-flanking region of the human MUC5AC mucin gene and transcriptional up-regulation by bacterial exoproducts. J Biol Chem 273, 6812-6820

18. Young HW, Williams OW, Chandra D et al (2007) Central role of Muc5ac expression in mucous metaplasia and its regulation by conserved 5' elements. Am J Respir Cell Mol Biol 37, 273-290

19. Yoon JH, Moon HJ, Seong JK et al (2002) Mucociliary differentiation according to time in human nasal epithelial cell culture. Differentiation 70, 77-83

20. Kim YJ, Cho HJ, Shin WC, Song HA, Yoon JH and Kim $\mathrm{CH}$ (2014) Hypoxia-Mediated Mechanism of MUC5AC Production in Human Nasal Epithelia and Its Implication in Rhinosinusitis. PLoS One 9, e98136

21. Miyamoto N, de Kozak Y, Normand N et al (2008) PIGF-1 and VEGFR-1 pathway regulation of the external epithelial hemato-ocular barrier. A model for retinal edema. Ophthalmic Res 40, 203-207

22. Ahdieh M, Vandenbos $T$ and Youakim A (2001) Lung epithelial barrier function and wound healing are decreased by IL-4 and IL-13 and enhanced by IFN-gamma. Am J Physiol Cell Physiol 281, C2029-2038

23. Sajjan U, Wang Q, Zhao Y, Gruenert DC and Hershenson MB (2008) Rhinovirus disrupts the barrier function of polarized airway epithelial cells. Am J Respir Crit Care Med 178, 1271-1281

24. Weis SM and Cheresh DA (2005) Pathophysiological consequences of VEGF-induced vascular permeability. Nature 437, 497-504

25. Aiello LP, Avery RL, Arrigg PG et al (1994) Vascular endothelial growth factor in ocular fluid of patients with diabetic retinopathy and other retinal disorders. $\mathrm{N}$ Engl J Med 331, 1480-1487

26. Nagashima M, Yoshino S, Ishiwata T and Asano G (1995)
Role of vascular endothelial growth factor in angiogenesis of rheumatoid arthritis. J Rheumatol 22, 1624-1630

27. Jung $\mathrm{HH}$, Kim MW, Lee JH et al (1999) Expression of vascular endothelial growth factor in otitis media. Acta Otolaryngol 119, 801-808

28. Matsune S, Ohori J, Sun D, Yoshifuku K, Fukuiwa T and Kurono Y (2008) Vascular endothelial growth factor produced in nasal glands of perennial allergic rhinitis. Am J Rhinol 22, 365-370

29. Lee YC, Kwak YG and Song CH (2002) Contribution of vascular endothelial growth factor to airway hyperresponsiveness and inflammation in a murine model of toluene diisocyanate-induced asthma. J Immunol 168, 3595-3600

30. Sun D, Matsune S, Ohori J, Fukuiwa T, Ushikai $M$ and Kurono Y (2005) TNF-alpha and endotoxin increase hypoxia-induced VEGF production by cultured human nasal fibroblasts in synergistic fashion. Auris Nasus Larynx 32, 243-249

31. Divekar RD, Samant S, Rank MA et al (2015) Immunological profiling in chronic rhinosinusitis with nasal polyps reveals distinct VEGF and GM-CSF signatures during symptomatic exacerbations. Clin Exp Allergy 45, 767-778

32. Song HA, Kim YS, Cho HJ et al (2017) Hypoxia Modulates Epithelial Permeability via Regulation of Vascular Endothelial Growth Factor in Airway Epithelia. Am J Respir Cell Mol Biol 57, 527-535

33. Takano K, Kojima T, Go M et al (2005) HLA-DR- and CD11c-positive dendritic cells penetrate beyond well-developed epithelial tight junctions in human nasal mucosa of allergic rhinitis. J Histochem Cytochem 53, 611-619

34. Nelson WJ (2003) Adaptation of core mechanisms to generate cell polarity. Nature $422,766-774$

35. Niessen CM (2007) Tight junctions/adherens junctions: basic structure and function. J Invest Dermatol 127, 2525-2532

36. Capaldo CT and Macara IG (2007) Depletion of E-cadherin disrupts establishment but not maintenance of cell junctions in Madin-Darby canine kidney epithelial cells. Mol Biol Cell 18, 189-200

37. Takeuchi $K$, Kishioka $C$, Ishinaga $H$, Sakakura $Y$ and Majima $Y$ (2001) Histamine alters gene expression in cultured human nasal epithelial cells. J Allergy Clin Immunol 107, 310-314

38. Jang YJ, Kim HG, Koo TW and Chung PS (2002) Localization of ZO-1 and E-cadherin in the nasal polyp epithelium. Eur Arch Otorhinolaryngol 259, 465-469

39. Yeo NK and Jang YJ (2010) Rhinovirus infection-induced alteration of tight junction and adherens junction components in human nasal epithelial cells. Laryngoscope 120, 346-352

40. Min HJ, Kim TH, Yoon JH and Kim CH (2015) Hypoxia increases epithelial permeability in human nasal epithelia. Yonsei Med J 56, 825-831

41. Lotze MT and Tracey KJ (2005) High-mobility group box 1 protein (HMGB1): nuclear weapon in the immune arsenal. Nat Rev Immunol 5, 331-342

42. Bonaldi T, Talamo F, Scaffidi P et al (2003) Monocytic 
cells hyperacetylate chromatin protein HMGB1 to redirect it towards secretion. EMBO J 22, 5551-5560

43. Hori O, Brett J, Slattery T et al (1995) The receptor for advanced glycation end products (RAGE) is a cellular binding site for amphoterin. Mediation of neurite outgrowth and co-expression of rage and amphoterin in the developing nervous system. J Biol Chem 270, 25752-25761

44. Tang D, Kang R, Livesey KM et al (2010) Endogenous HMGB1 regulates autophagy. J Cell Biol 190, 881-892

45. Han SJ, Min HJ, Yoon SC et al (2015) HMGB1 in the pathogenesis of ultraviolet-induced ocular surface inflammation. Cell Death Dis 6, e1863

46. Min HJ, Kim SJ, Kim TH, Chung HJ, Yoon JH and Kim CH (2015) Level of secreted HMGB1 correlates with severity of inflammation in chronic rhinosinusitis. Laryngoscope 125, E225-230

47. Itoh T, Iwahashi S, Shimoda M et al (2011) High-mobility group box 1 expressions in hypoxia-induced damaged mouse islets. Transplant Proc 43, 3156-3160

48. Hamada T, Torikai M, Kuwazuru A et al (2008) Extracellular high mobility group box chromosomal protein 1 is a coupling factor for hypoxia and inflammation in arthritis. Arthritis Rheum 58, 2675-2685

49. Min HJ, Kim JH, Yoo JE et al (2017) ROS-dependent HMGB1 secretion upregulates IL-8 in upper airway epithelial cells under hypoxic condition. Mucosal Immunol 10, 685-694

50. Desireddi JR, Farrow KN, Marks JD, Waypa GB and Schumacker PT (2010) Hypoxia increases ROS signaling and cytosolic $\mathrm{Ca}(2+)$ in pulmonary artery smooth muscle cells of mouse lungs slices. Antioxid Redox Signal 12, 595-602

51. Joo JH, Ryu JH, Kim CH et al (2012) Dual oxidase 2 is essential for the toll-like receptor 5-mediated inflammatory response in airway mucosa. Antioxid Redox Signal $16,57-70$

52. Riechelmann $H$, Deutschle T, Friemel E, Gross $H J$ and Bachem M (2003) Biological markers in nasal secretions. Eur Respir J 21, 600-605

53. Riechelmann H, Deutschle T, Rozsasi A, Keck T, Polzehl $\mathrm{D}$ and Burner $\mathrm{H}$ (2005) Nasal biomarker profiles in acute and chronic rhinosinusitis. Clin Exp Allergy 35, 1186-1191

54. Kramer MF, Burow G, Pfrogner E and Rasp G (2004) In vitro diagnosis of chronic nasal inflammation. Clin Exp Allergy 34, 1086-1092

55. Deroee AF, Naraghi $M$, Sontou AF, Ebrahimkhani MR and Dehpour AR (2009) Nitric oxide metabolites as biomarkers for follow-up after chronic rhinosinusitis surgery. Am J Rhinol Allergy 23, 159-161

56. Shimizu S, Kouzaki $H$, Kato T, Tojima I and Shimizu T (2016) HMGB1-TLR4 signaling contributes to the secretion of interleukin 6 and interleukin 8 by nasal epithelial cells. Am J Rhinol Allergy 30, 167-172

57. Paris G, Pozharskaya T, Asempa T and Lane AP (2014) Damage-associated molecular patterns stimulate interleukin-33 expression in nasal polyp epithelial cells. Int Forum Allergy Rhinol 4, 15-21

58. Yang $\mathrm{H}$, Wang $\mathrm{H}$, Czura $\mathrm{CJ}$ and Tracey KJ (2005) The cytokine activity of HMGB1. J Leukoc Biol 78, 1-8

59. Lv B, Wang H, Tang Y, Fan Z, Xiao X and Chen F (2009) High-mobility group box 1 protein induces tissue factor expression in vascular endothelial cells via activation of NF-kappaB and Egr-1. Thromb Haemost 102, 352-359

60. Fiuza C, Bustin M, Talwar S et al (2003) Inflammationpromoting activity of HMGB1 on human microvascular endothelial cells. Blood 101, 2652-2660 